16

\title{
Спиновый угловой момент нелинейной поверхностной волны на границе раздела обычного и топологического изоляторов
}

\author{
(C) А.И. Маймистов ${ }^{1}$, Е.И. Ляшко ${ }^{2}$ \\ ${ }^{1}$ Национальный исследовательский ядерный университет МИФИ, \\ 115409 Москва, Россия \\ ${ }^{2}$ Московский фризико-технический институт, \\ 141700 Долгопрудный, Россия \\ e-mail: aimaimistov@gmail.com
}

Поступила в редакцию 11.11.2018 г.

В окончательной редакции 27.12.2018 г.

Принята к публикации 09.01.2019 г.

Рассмотрены поверхностные волны, распространяющиеся вдоль границы раздела диэлектрика, обладающего нелинейной восприимчивостью третьего порядка и топологического изолятора. Оптическая нелинейность диэлектрика обеспечивает существование поверхностной волны. Для диэлектриков с положительной или отрицательной линейной диэлектрической проницаемостью определена плотность спинового углового момента поверхностной волны. Показано, что вектор спинового углового момента имеет проекцию на нормаль к поверхности раздела, что составляет отличие от случая обычных поверхностных поляритонов или плазмонполяритонов. Дискретный характер топологического числа проявляется в дискретности значений нормальной и касательной компонент плотности спинового углового момента. Рост напряженности электрического поля волны на границе раздела сред изменяет величину спинового углового момента и может привести к его исчезновению.

DOI: $10.21883 /$ OS.2019.05.47656.356-18

\section{Введение}

Угловой момент, переносимый электромагнитной волной, является характеристикой поля, которая обычно меньше привлекала внимание, нежели импульс поля или поток энергии. Возникший интерес к формированию пучков света с фазовыми дефектами волнового фронта излучения, изучение возможности передачи момента вращения от поля к материальным телам и создание оптических манипуляторов для задач нанотехнологии возродил внимание к этой важной характеристике электромагнитного поля. В интегральной оптике и плазмонике роль пучков играют поверхностные волны. По этой причине привлекают внимание различные характеристики поверхностной волны, в частности угловой момент. Полный угловой момент электромагнитного поля $\mathbf{J}\left(\mathbf{r}_{0}\right)$ относительно точки с радиусом-вектором $\mathbf{r}_{0}$ можно представить в виде суммы $\mathbf{J}\left(\mathbf{r}_{0}\right)=\mathbf{L}\left(\mathbf{r}_{0}\right)+\mathbf{S}_{\mathrm{in}}$, в которой первое слагаемое зависит от вектора $\mathbf{r}_{0}$. Вектор $\mathbf{L}\left(\mathbf{r}_{0}\right)$ называется орбитальным угловым моментом поля или моментом импульса. Второе слагаемое характеризует внутренние свойства самого электромагнитного поля и называется спиновым угловым моментом поля [1-5].

В однородном изотропном пространстве спиновый угловой момент складывается из спиральностей фотонов. Если число фотонов, имеющих различные спиральности, одинаково, то волна линейно поляризована и спинового момента не несет. В противном случае волна будет эллиптически поляризована, и такая волна несет спино- вый момент ${ }^{1}$. В пространственно неоднородной среде, где фронт волны не плоский, волна может обладать орбитальным и спиновым моментами.

Простейший случай неоднородной среды представляет граница раздела двух однородных изотропных сред. При определенных условиях вдоль границы раздела может бежать поверхностная волна, у которой могут быть продольные и поперечные компоненты напряженностей электрического и магнитного полей. Аналогичная ситуация имеет место в планарных (или в двумерных) волноводах [6]. В этих случаях направленные волны обладают спиновым моментом [7,8]. Исследованию полного углового момента поверхностных волн большое внимание уделялось в работах [8-13]. Волны, поляризованные перпендикулярно направлению распространения (ТЕ-волны), обладают только угловым орбитальным моментом. Для случая поверхностной ТМ-волны, распространяющейся вдоль поверхности раздела вакуума и среды с отрицательной диэлектрической проницаемостью, спиновый момент ненулевой. Как показано в [8,9], спиновый угловой момент направлен ортогонально волновому вектору поверхностной волны и лежит в плоскости раздела сред.

В приближении линейной оптики поверхностные волны ТМ-типа существуют, если диэлектрическая проницаемость одной из сред отрицательная. Это условие выполняется для поверхности металла, и тогда говорят о

\footnotetext{
1 Далее для краткости будет использоваться термин спиновый момент вместо спиновый угловой момент.
} 
поверхностном плазмон-поляритоне $[12,13]$. Отрицательная диэлектрическая проницаемость может быть получена в среде с диэлектрической проницаемостью, близкой к нулю [14-19]. Также отрицательной диэлектрической проницаемостью обладают гиперболические метаматериалы [20,21]. Топологический изолятор (ТИ) [22,23] характеризуется поверхностным магнитоэлектрическим эффектом, который приводит к повороту векторов напряженностей магнитного и электрического полей при пересечении границы раздела обычного диэлектрика и ТИ. По этой причине поверхностные волны содержат все три компоненты электрического и магнитного полей. Такого рода поверхностные волны были рассмотрены в [24-29].

В данной работе будет рассмотрена граница раздела ТИ и нелинейной среды. Материалы, обладающие нелинейной восприимчивостью, есть среди сред с диэлектрической проницаемостью, близкой к нулю [19,30,31]. Нелинейную восприимчивость керровского типа могут иметь металлы [32-36]. На границе раздела диэлектрика с нелинейной восприимчивостью третьего порядка и ТИ может существовать поверхностная волна, являющаяся суперпозицией поперечной электрической (ТЕ) и поперечной магнитной (ТМ) волн, если переносимая ею мощность превышает определенное пороговое значение [27]. Для вычисления углового спинового момента поверхностной волны будут использованы полученные в [27] результаты. Присутствие ТИ приводит к тому, что вектор плотности спинового момента направлен нормально к направлению распространения и имеет как нормальную, так и касательную проекции. Результатом дискретности топологического числа, характеризующего ТИ, является дискретность проекций плотности спинового момента. Максимальное значение спинового момента зависит от интенсивности электрической волны на поверхности раздела сред, что обусловлено нелинейной восприимчивостью диэлектрика.

\section{Поперечные распределения электрических полей поверхностной волны}

Рассматривается плоская граница раздела топологического диэлектрика и обычного диэлектрика, который характеризуется кубической (керровской) нелинейностью (нелинейной восприимчивостью третьего порядка). Пусть ось координат $X$ направлена нормально к поверхности раздела двух сред. Оси $Y$ и $Z$ лежат в плоскости раздела. Ось $Z$ выбрана вдоль вектора постоянной распространения поверхностной волны. В такой системе координат из-за планарной симметрии напряженности всех полей зависят только от переменных $x, z$ и времени [6]. Для компонент напряженности электрического поля $E_{y}$ и $E_{z}$ можно записать волновые уравнения, а остальные компоненты выразить через $E_{y}$ и $E_{z}$. Уравнения Максвелла приводятся к системе уравнений для компонент Фурье напряженностей полей, зависящих от пространственных переменных $x, z$ и частоты $\omega$ :

$$
\begin{gathered}
\frac{\partial^{2} E_{y}}{\partial x^{2}}+\frac{\partial^{2} E_{y}}{\partial z^{2}}+k_{0}^{2} \mu D_{y}=0 \\
H_{z}=-\frac{i}{k_{0} \mu} \frac{\partial E_{y}}{\partial x}, \quad H_{x}=\frac{i}{k_{0} \mu} \frac{\partial E_{y}}{\partial z}, \\
\frac{\partial E_{x}}{\partial z}-\frac{\partial E_{z}}{\partial x}=i k_{0} \mu H_{y}, \quad D_{z}=\frac{i}{k_{0}} \frac{\partial H_{y}}{\partial x}, \quad D_{x}=-\frac{i}{k_{0}} \frac{\partial H_{y}}{\partial z},
\end{gathered}
$$

где $k_{0}=\omega / c$. Из-за однородности среды вдоль оси $z$ напряженности полей и индукция могут быть представлены в следующем виде: $\mathbf{E}(x, z ; \omega)=\mathbf{e}(x) \exp (i \beta z)$, $\mathbf{H}(z, x ; \omega)=\mathbf{h}(x) \exp (i \beta z)$ и $\mathbf{D}(x, z ; \omega)=\mathbf{d} \exp (i \beta z)$, где $\beta$ - постоянная распространения [6]. Это позволяет свести систему приведенных выше уравнений к двум обыкновенным уравнениям относительно $e_{y}(x, \omega)$ и $e_{z}(x, \omega)$. Остальные компоненты выражаются через $e_{y}(x, \omega)$ и $e_{z}(x, \omega)$. Векторный потенциал $\mathbf{A}(x, z ; \omega)=\mathbf{a}(x ; \omega) \exp (i \beta z)$ выбирается в кулоновской калибровке: $\operatorname{div} \mathbf{A}=0$. Его амплитуда связана с электрическим полем в этом случае соотношением $\mathbf{e}(x)=i k_{0} \mathbf{a}(x)$. В дальнейшем зависимость от частоты $\omega$ можно не указывать.

Надо заметить, что волновые уравнения, определяющие $e_{y}(x)$ и $e_{z}(x)$, не содержат комплексных величин (потерями полностью пренебрегаем). Следовательно, если $e_{y, z}(x)$ есть решения соответствующих уравнений, то и $e^{i \psi_{1}} e_{y}(x)$ и $e^{i \psi_{2}} e_{z}(x)$ тоже являются решениями при произвольных постоянных фазах $\psi_{1}$ и $\psi_{2}$. Однако условия связи амплитуд полей при $x=0$ фиксируют разность этих фаз. Поскольку распределения электрического и магнитного полей и дисперсионное соотношение были получены ранее [27-29], здесь можно использовать выражения, которые нужны в данном исследовании для вычисления плотности спинового момента поверхностной волны.

Поперечные распределения напряженностей электрических полей и векторного потенциала имеют следующий вид. В области $x<0$ (ТИ)

$$
\begin{gathered}
e_{x}^{(1)}=(\beta / i q) B e^{q x}, \quad e_{y}^{(1)}=A e^{q x}, \quad e_{z}^{(1)}=B e^{q x}, \\
a_{x}^{(1)}=-\left(\beta / k_{0} q\right) B e^{q x}, \quad a_{y}^{(1)}=\left(1 / i k_{0}\right) A e^{q x}, \\
a_{z}^{(1)}=\left(1 / i k_{0}\right) B e^{q x},
\end{gathered}
$$

где $q^{2}=\beta^{2}-k_{0}^{2} \varepsilon_{1}>0$. Постоянные фазы входят в определение амплитуд $A$ и $B$, которые считаются комплексными величинами. В области $x>0$ (нелинейная обычная среда)

$$
\begin{gathered}
e_{y}^{(2)}=\frac{A_{0} \cosh \left(p x_{0}\right)}{\cosh \left[p\left(x-x_{0}\right)\right]}, \quad e_{z}^{(2)}=\frac{B_{0} \sinh \left(p x_{0}\right)}{\sinh \left[p\left(x-x_{0}\right)\right]}, \\
e_{x}^{(2)}=\frac{\beta}{i p^{2}} \frac{\partial e_{z}^{(2)}}{\partial x},
\end{gathered}
$$




$$
\begin{gathered}
a_{y}^{(2)}=\frac{A_{0} \cosh \left(p x_{0}\right)}{i k_{0} \cosh \left[p\left(x-x_{0}\right)\right]}, \quad a_{z}^{(2)}=\frac{B_{0} \sinh \left(p x_{0}\right)}{i k_{0} \sinh \left[p\left(x-x_{0}\right)\right]}, \\
a_{x}^{(2)}=\frac{\beta}{i p^{2}} \frac{\partial a_{z}^{(2)}}{\partial x}
\end{gathered}
$$

где $p^{2}=\beta^{2}-k_{0}^{2} \varepsilon_{2}>0, x_{0}-$ положение максимума напряженности компоненты электрического поля $e_{y}(x)$, а комплексные амплитуды $A_{0}, B_{0}$ определены как

$$
A_{0}=\sqrt{\frac{2 p^{2}}{k_{0}^{2}\left|\varepsilon_{k}\right|}} \frac{e^{i \psi_{1}}}{\cosh \left(p x_{0}\right)}, \quad B_{0}=\sqrt{\frac{2 p^{2}}{k_{0}^{2}\left|\varepsilon_{k}\right|}} \frac{( \pm 1) e^{i \psi_{2}}}{\sinh \left(p x_{0}\right)}
$$

Существенными переменными являются $e_{y}(x)$ и $e_{z}(x)$. Поэтому необходимо определить как решения $e_{y, z}^{(1)}(x)(1)$ переходят в $e_{y, z}^{(2)}(x)$ (3) при $x=0$. Воспользовавшись выражениями, которые связывают напряженности и индукции при $x=0[22,23,27]$, можно получить условия непрерывности касательных проекций напряженностей электрических полей и разности производных для $e_{y}(x)$ и $e_{z}(x)$ в точке $x=0$ :

$$
\begin{gathered}
e_{y}^{(1)}(0)=e_{y}^{(2)}(0), \quad e_{z}^{(1)}(0)=e_{z}^{(2)}(0), \\
\frac{\partial}{\partial x} e_{y}^{(1)}(0)-\frac{\partial}{\partial x} e_{y}^{(2)}(0)=i k_{0} \kappa e_{z}^{(1)}(0), \\
\frac{\varepsilon_{1}}{q^{2}} \frac{\partial}{\partial x} e_{z}^{(1)}(0)-\frac{\varepsilon_{2}}{p^{2}} \frac{\partial}{\partial x} e_{z}^{(2)}(0)=i \frac{\kappa}{k_{0}} e_{y}^{(1)}(0),
\end{gathered}
$$

где $\kappa=\left(e^{2} / 4 \pi c \hbar\right) \Delta \theta$. Параметр $\kappa$ содержит разность топологических чисел $\Delta \theta=\theta^{(1)}-\theta^{(2)}$, которые характеризуют первую и вторую среды [22]. В рассматриваемом случае $\theta^{(1)}=1+2 n, n-$ целое число, и $\theta^{(2)}=0$.

Непрерывность касательных проекций напряженностей электрических полей при $x=0$ означает, что $A-A_{0}=0$ и $B-B_{0}=0$. Так что $\arg A=\psi_{1}$ и $\arg B=\psi_{2}$. Используя (3) и два последних уравнения из (5), можно записать

$\left(q+p \tanh p x_{0}\right) A=i \kappa k_{0} B, \quad\left(\frac{\varepsilon_{1}}{q}+\frac{\varepsilon_{2}}{p \tanh p x_{0}}\right) B=i \frac{\kappa}{k_{0}} A$.

Далее будет использовано обозначение $p \tanh p x_{0}=\tilde{p}$. Первое уравнение из (6) можно переписать как $(q+\tilde{p})|A|=i \kappa k_{0}|B| \exp \left(i \psi_{2}-i \psi_{1}\right)$, откуда следует, что $\psi_{1}-\psi_{2}=\pi / 2$. Система уравнений (6) приводит к дисперсионному соотношению

$$
(q+\tilde{p})\left(\varepsilon_{2} q+\varepsilon_{1} \tilde{p}\right)+\kappa^{2} \tilde{p} q=0,
$$

которое эквивалентно уравнению (24) из [27]. Это уравнение имеет решения, когда $\varepsilon_{1}>0, \varepsilon_{2}<0$ или $\varepsilon_{1}>0$, $\varepsilon_{2}>0$, но $\tilde{p}<0$. Первый случай может реализоваться на границе раздела ТИ и металла [25], ТИ и гиперболического метаматериала $[28,29]$ или ТИ и среды с показателем преломления, близким к нулю. Второй случай отвечает нелинейной поверхностной волне [27]. Параметр $x_{0}$ возникает как постоянная интегрирования при решении волнового уравнения для $e_{z}(x)$ в нелинейной среде. Чтобы решение не обращалось в бесконечность в области, занимаемой нелинейной средой, необходимо выбрать $x_{0}<0$. Отсюда следует, что $\tilde{p}<0$. Максимум напряженности электрического поля достигается на границе раздела сред.

\section{Вычисление спинового углового момента}

Спиновый момент определяется [1-3] выражением

$$
\mathbf{S}_{i n}=(4 \pi c)^{-1} \int \mathbf{E} \times \mathbf{A} d V .
$$

Если ввести вектор $\mathbf{g}=2 \operatorname{Re}\left(\mathbf{e} \times \mathbf{a}^{*}\right)$, то усредненный по быстрым колебаниям во времени спиновый момент есть $\left\langle\mathbf{S}_{i n}\right\rangle=(4 \pi c)^{-1} \int \mathbf{g} d V$. Используя формулы (1)(4), можно получить выражения для компонент вектора плотности спинового момента g. В области $x<0$

$$
\begin{gathered}
g_{x}^{(1)}=\frac{2 i}{k_{0}}\left(A B^{*}-A^{*} B\right) e^{2 q x}, \quad g_{y}^{(1)}=-\frac{4 \beta}{k_{0} q}|B|^{2} e^{2 q x}, \\
g_{z}^{(1)}=\frac{2 \beta}{k_{0} q}\left(A B^{*}+A^{*} B\right) e^{2 q x} .
\end{gathered}
$$

В области $x>0$

$$
\begin{gathered}
g_{x}^{(2)}=\frac{2 i}{k_{0}}\left(A_{0} B_{0}^{*}-A_{0}^{*} B_{0}\right) \frac{\sinh \left(2 p x_{0}\right)}{\sinh \left[2 p\left(x-x_{0}\right)\right]}, \\
g_{y}^{(2)}=\frac{4 \beta\left|B_{0}\right|^{2}}{k_{0} p} \frac{\sinh ^{2}\left(p x_{0}\right) \cosh \left[p\left(x-x_{0}\right)\right]}{\sinh ^{3}\left[p\left(x-x_{0}\right)\right]}, \\
g_{z}^{(2)}=-\frac{\beta}{k_{0} p}\left(A_{0} B_{0}^{*}+A_{0}^{*} B_{0}\right) \frac{\sinh \left(2 p x_{0}\right)}{\sinh ^{2}\left[p\left(x-x_{0}\right)\right]} .
\end{gathered}
$$

Из уравнений (6) с учетом того, что $A-A_{0}=0$, $B-B_{0}=0$, можно найти связь между касательными компонентами напряженности электрического поля при $x=0$ :

$$
B=\frac{i \kappa q \tilde{p}}{k_{0}\left(\tilde{p} \varepsilon_{1}+q \varepsilon_{2}\right)} A \equiv i \rho A .
$$

С учетом (10) выражения для компонент плотности спинового момента $\mathbf{g}$ записываются как

$$
\begin{gathered}
g_{x}^{(1)}=\frac{4 \rho}{k_{0}}|A|^{2} e^{2 q x} \\
g_{y}^{(1)}=-\frac{4 \beta \rho^{2}}{k_{0} q}|A|^{2} e^{2 q x}, \quad g_{z}^{(1)}=0 \\
g_{x}^{(2)}=\frac{4 \rho}{k_{0}} \frac{|A|^{2} \sinh \left(2 p x_{0}\right)}{\sinh \left[2 p\left(x-x_{0}\right)\right]}, \\
g_{y}^{(2)}=\frac{4 \beta \rho^{2}}{k_{0} p} \frac{|A|^{2} \sinh ^{2}\left(p x_{0}\right) \cosh \left[p\left(x-x_{0}\right)\right]}{\sinh ^{3}\left[p\left(x-x_{0}\right)\right]}, \quad g_{z}^{(2)}=0 .
\end{gathered}
$$

Как видно из (13), нормальная проекция вектора спинового момента не равна нулю в отличие от случая поверхностных плазмон-поляритонов $[9,10]$. На границе раздела 
обычных сред (для которых $\theta^{(1)}=\theta^{(2)}=0$ ) волны ТЕ и ТМ независимы. Система уравнений (6) распадается на два несвязанных уравнения. Для ТМ-волны $A=A_{0}=0$, но $B=B_{0} \neq 0$ при выполнении дисперсионного соотношения $\left(\varepsilon_{2} q+\varepsilon_{1} \tilde{p}\right)=0$. Из (12) и (13) сразу следует, что в таком случае $g_{x}^{(1)}=g_{x}^{(2)}=0$. Для ТЕ-волны $A=A_{0} \neq 0$, но $B=B_{0}=0$. Из (12) и (13) следует, что в этом случае все компоненты векторов $\mathbf{g}^{(1)}$ и $\mathbf{g}^{(2)}$ равны нулю. Таким образом, при выполнении условия $\theta^{(1)}=\theta^{(2)}=0$ воспроизводятся результаты работ [8-11].

\section{Роль топологического числа в величине спинового момента}

Максимальное значение направленной по нормали к границе раздела компоненты вектора плотности спинового момента $g_{x}^{(1)}(0)=g_{x}^{(2)}(0)=g_{x}(0)$ дается выражениeM

$$
g_{x}(0)=\frac{4 \rho}{k_{0}}|A|^{2}, \quad \text { где } \rho=\frac{\kappa q \tilde{p}}{k_{0}\left(\tilde{p} \varepsilon_{1}+q \varepsilon_{2}\right)} .
$$

Эффект керровской нелинейности заключается [27] в зависимости параметра $\tilde{p}$ от интенсивности электрической волны на границе раздела:

$$
\tilde{p}^{2}=\beta^{2}-k_{0}^{2}\left(\varepsilon_{2}+\varepsilon_{K}|A|^{2} / 2\right) .
$$

Ограниченность амплитуды напряженности электрического поля требует выбирать знак минус при извлечении квадратного корня из $\tilde{p}^{2}$, т.е. следует положить $\tilde{p}<0$. Таким образом, дисперсионное соотношение выглядит как

$$
(q-|\tilde{p}|)\left(\varepsilon_{2} q-\varepsilon_{1}|\tilde{p}|\right)-\kappa^{2}|\tilde{p}| q=0 .
$$

Имеют место два случая выбора знака параметра $\varepsilon_{2}$ : $\varepsilon_{2}>0$ и $\varepsilon_{2}<0$. Для материалов с диэлектрической проницаемостью, близкой к нулю [19,30,31], оба случая могут быть реализованы путем подбора частоты излучения.

\section{Случай положительной диэлектрической проницаемости}

Из дисперсионного соотношения

$$
(q-|\tilde{p}|)\left(\varepsilon_{2} q-\varepsilon_{1}|\tilde{p}|\right)-\kappa^{2}|\tilde{p}| q=0
$$

для переменной $\tau=|\tilde{p}| / q \varepsilon_{2}$ следует уравнение

$$
\tau^{2}-\left(\frac{1}{\varepsilon_{1}}+\frac{1}{\varepsilon_{2}}+\frac{\kappa^{2}}{\varepsilon_{1} \varepsilon_{2}}\right) \tau+\frac{1}{\varepsilon_{1} \varepsilon_{2}}=0 .
$$

Корни этого уравнения $\tau_{ \pm}(\kappa)$ даются выражениями

$$
\begin{gathered}
2 \tau_{ \pm}(\kappa)=R \pm \sqrt{R^{2}-\frac{4}{\varepsilon_{1} \varepsilon_{2}}}, \\
R=\left(\frac{1}{\varepsilon_{1}}+\frac{1}{\varepsilon_{2}}+\frac{\kappa^{2}}{\varepsilon_{1} \varepsilon_{2}}\right)
\end{gathered}
$$

При $\kappa=0$ (граница раздела обычных сред с $\theta=0$ или ТИ с одинаковыми топологическими числами $\theta$ ) отсюда следует, что $\tau_{+}(0)=\varepsilon_{1}^{-1}, \tau_{-}(0)=\varepsilon_{2}^{-1}$.

Полагая $|\tilde{p}|=q \varepsilon_{2} \tau_{+}(0)$ и используя явные выражения для параметров $|\tilde{p}|$ и $q$, можно найти зависимость постоянной распространения $\beta$ от частоты и амплитуды поля на границе раздела:

$$
\beta^{2}=k_{0}^{2}\left[\frac{\varepsilon_{1} \varepsilon_{2}}{\varepsilon_{1}+\varepsilon_{2}}-\frac{\varepsilon_{K} \varepsilon_{1}^{2}}{\varepsilon_{1}^{2}-\varepsilon_{2}^{2}}|A|^{2}\right] .
$$

Полагая $|\tilde{p}|=q \varepsilon_{2} \tau_{-}(0)$, можно найти дисперсионное соотношение в виде $|\tilde{p}|=q$, которое не имеет решения и, следовательно, корень $\tau_{-}(0)$ неприемлем.

Параметр $\kappa^{2}$ примерно равен $10^{-4} \Delta \theta$. Если $\Delta \theta$ порядка единицы или сотни, можно считать $\kappa^{2} \ll 1$ и разложить $\tau_{ \pm}(\kappa)$ по степеням $\kappa$ до $\kappa^{2}$. Выражение, стоящее под радикалом в (16), далее обозначенное как $D^{2}$, принимает следующий вид:

$$
D^{2}=\left(\frac{1}{\varepsilon_{1}}-\frac{1}{\varepsilon_{2}}\right)^{2}\left(1+\frac{2 \kappa^{2}\left(\varepsilon_{1}+\varepsilon_{2}\right)}{\left(\varepsilon_{1}-\varepsilon_{2}\right)^{2}}\right)+O\left(\kappa^{4}\right) .
$$

Используя это выражение, из (16) можно найти

$$
\begin{aligned}
& \tau_{+}(\kappa)=\frac{1}{\varepsilon_{1}}\left(1+\frac{\kappa^{2}}{\varepsilon_{2}-\varepsilon_{1}}\right)+O\left(\kappa^{4}\right), \\
& \tau_{-}(\kappa)=\frac{1}{\varepsilon_{2}}\left(1-\frac{\kappa^{2}}{\varepsilon_{2}-\varepsilon_{1}}\right)+O\left(\kappa^{4}\right) .
\end{aligned}
$$

Входящий в (14) множитель $\rho$ есть

$$
\rho=\frac{\kappa q|\tilde{p}|}{k_{0}\left(|\tilde{p}| \varepsilon_{1}-q \varepsilon_{2}\right)}=\frac{|\tilde{p}|-q}{k_{0} \kappa}=\frac{|\tilde{p}|}{k_{0} \kappa}\left(1-\frac{1}{\varepsilon_{2} \tau_{ \pm}}\right) .
$$

Для $\tau$ вычисление $\rho$ даст

$$
\rho=\frac{|\tilde{p}|}{k_{0}}\left[\left(1-\frac{\varepsilon_{1}}{\varepsilon_{2}}\right) \kappa^{-1}+\frac{\varepsilon_{1}}{\varepsilon_{2}\left(\varepsilon_{2}-\varepsilon_{1}\right)} \kappa\right]+O\left(\kappa^{3}\right) .
$$

Таким образом,

$$
g_{x}(0) \approx \frac{4|\tilde{p}||A|^{2}}{k_{0}^{2}}\left[\left(1-\frac{\varepsilon_{1}}{\varepsilon_{2}}\right) \kappa^{-1}+\frac{\varepsilon_{1}}{\varepsilon_{2}\left(\varepsilon_{2}-\varepsilon_{1}\right)} \kappa\right] .
$$

Надо заметить, что величина $\kappa$ пропорциональна топологическому числу $\theta^{(1)}=1+2 n$, где $n-$ целое число. Следовательно, присутствие ТИ проявляется в дискретности нормальной компоненты плотности спинового момента:

$$
\begin{aligned}
& g_{x}(0) \approx \frac{4|\tilde{p}||A|^{2}}{k_{0}^{2}}\left(1-\frac{\varepsilon_{1}}{\varepsilon_{2}}\right)\left(\frac{4 \pi c \hbar}{e^{2}(1+2 n)}\right) \\
& +\frac{|\tilde{p}||A|^{2} \varepsilon_{1}}{k_{0} \varepsilon_{2}\left(\varepsilon_{2}-\varepsilon_{1}\right)}\left(\frac{e^{2}}{\pi c \hbar}\right)(1+2 n), \quad n=0,1,2, \ldots
\end{aligned}
$$


Для тангенциальной компоненты плотности спинового момента $g_{y}^{(1)}(0)$ аналогичным образом можно получить выражение

$$
\begin{aligned}
g_{y}^{(1)}(0) \approx & -\frac{4 \beta \tilde{p}^{2}|A|^{2}}{q k_{0}^{2}}\left[\left(1-\frac{\varepsilon_{1}}{\varepsilon_{2}}\right)^{2}\left(\frac{4 \pi c \hbar}{e^{2}(1+2 n)}\right)^{2}\right. \\
& \left.+\left(1-\frac{\varepsilon_{1}}{\varepsilon_{2}}\right) \frac{\varepsilon_{1}}{\varepsilon_{2}\left(\varepsilon_{2}-\varepsilon_{1}\right)}\right] .
\end{aligned}
$$

В этом выражении $\tilde{p}^{2}=\beta^{2}-k_{0}^{2}\left(\varepsilon_{2}+\varepsilon_{K}|A|^{2} / 2\right)$.

\section{Случай отрицательной диэлектрической проницаемости $\varepsilon_{2}$}

Дисперсионное соотношение теперь имеет вид

$$
(q-|\tilde{p}|)\left(\left|\varepsilon_{2}\right| q+\varepsilon_{1}|\tilde{p}|\right)+\kappa^{2}|\tilde{p}| q=0 .
$$

Отсюда для переменной $\tau=|\tilde{p}| / q\left|\varepsilon_{2}\right|$ следует уравнение

$$
\tau^{2}-\left(\frac{1}{\left|\varepsilon_{2}\right|}-\frac{1}{\varepsilon_{1}}+\frac{\kappa^{2}}{\varepsilon_{1}\left|\varepsilon_{2}\right|}\right) \tau-\frac{1}{\varepsilon_{1}\left|\varepsilon_{2}\right|}=0 .
$$

Корни этого уравнения $\tau_{ \pm}(\kappa)$ даются выражениями

$$
\begin{gathered}
2 \tau_{ \pm}(\kappa)=R \pm \sqrt{R^{2}+\frac{4}{\varepsilon_{1}\left|\varepsilon_{2}\right|}}, \\
R=\left(\frac{1}{\left|\varepsilon_{2}\right|}-\frac{1}{\varepsilon_{1}}+\frac{\kappa^{2}}{\varepsilon_{1}\left|\varepsilon_{2}\right|}\right) .
\end{gathered}
$$

С учетом малости параметра $\kappa^{2}$ отсюда следует, что

$$
\begin{aligned}
& \tau_{+}(\kappa)=\frac{1}{\left|\varepsilon_{2}\right|}\left(1+\frac{\kappa^{2}}{\left|\varepsilon_{2}\right|+\varepsilon_{1}}\right)+O\left(\kappa^{4}\right), \\
& \tau_{-}(\kappa)=-\frac{1}{\varepsilon_{1}}\left(1-\frac{\kappa^{2}}{\left|\varepsilon_{2}\right|+\varepsilon_{1}}\right)+O\left(\kappa^{4}\right) .
\end{aligned}
$$

Второй корень надо отбросить, так как по определению $\tau(\kappa)>0$. Вычисление множителя $\rho$ в (14) дает

$$
\rho=\frac{|\tilde{\rho}|}{k_{0}} \frac{\kappa}{\left(\left|\varepsilon_{2}\right|+\varepsilon_{1}\right)}+O\left(\kappa^{3}\right) .
$$

Следовательно

$$
\begin{aligned}
g_{x}(0) & \approx \frac{4|\tilde{p}||A|^{2}}{k_{0}^{2}} \frac{\kappa}{\left(\left|\varepsilon_{2}\right|+\varepsilon_{1}\right)} \\
& =\frac{4|\tilde{p}||A|^{2}}{k_{0}^{2}\left(\left|\varepsilon_{2}\right|+\varepsilon_{1}\right)}\left(\frac{e^{2}}{4 \pi c \hbar}\right)(1+2 n), \quad n=0,1,2, \ldots
\end{aligned}
$$

Касательная компонента плотности спинового момента дается следующим выражением:

$$
\begin{aligned}
g_{y}^{(1)}(0) & =-\frac{4 \beta|\tilde{p}||A|^{2}}{k_{0}^{3}} \frac{\kappa^{2}}{\left(\left|\varepsilon_{2}\right|+\varepsilon_{1}\right)^{2}}+O\left(\kappa^{2}\right) \\
& \approx-\frac{4 \beta|\tilde{p}||A|^{2}}{k_{0}^{3}\left(\left|\varepsilon_{2}\right|+\varepsilon_{1}\right)^{2}}\left(\frac{e^{2}}{4 \pi c \hbar}\right)^{2}(1+2 n)^{2},
\end{aligned}
$$

$$
\begin{aligned}
g_{y}^{(2)}(0) & =\frac{4 \beta|\tilde{p}||A|^{2}}{k_{0}^{3}} \frac{\kappa^{2}}{\left(\left|\varepsilon_{2}\right|+\varepsilon_{1}^{2}\right)}+O\left(\kappa^{2}\right) \\
& \approx \frac{4 \beta|\tilde{p}||A|^{2}}{k_{0}^{3}\left(\left|\varepsilon_{2}\right|+\varepsilon_{1}\right)^{2}}\left(\frac{e^{2}}{4 \pi c \hbar}\right)^{2}(1+2 n)^{2} .
\end{aligned}
$$

Поскольку в определение касательной компоненты плотности спинового момента входит переменная $e_{x}$, которая не является непрерывной величиной в точке $x=0$, компонента $g_{y}$ разрывна при $x=0$.

\section{Заключение}

В данной работе рассмотрена поверхностная волна и вычислен ее спиновый угловой момент, который является внутренней характеристикой электромагнитного поля волны. Рассматривалась граница раздела между ТИ и обычным диэлектриком, для которого диэлектрическая проницаемость может быть положительной или отрицательной величиной. В отличие от результатов, полученных в [7-9], вектор плотности спинового углового момента не лежит полностью в плоскости границы раздела сред. Появление нормальной компоненты спинового углового момента обусловлено свойствами ТИ. Характерный для этого материала магнитоэлектрический эффект приводит к изменению условий непрерывности для нормальных компонент электрической индукции и касательных компонент магнитной индукции. Поляризация волны при переходе через границу раздела меняется как в случае эффекта Фарадея. Если возможно образование поверхностной волны, то у нее все компоненты напряженности электрического поля оказываются ненулевыми. Следствием является поперечный спиновый момент с ненулевой нормальной компонентой.

Благодаря нелинейным свойствам диэлектрика существование поверхностной волны не требует отрицательности линейной диэлектрической проницаемости. Здесь рассматривалась упрощенная модель нелинейного диэлектрика, предложенная в работе [37] и использованная для анализа нелинейных поверхностных волн на границе раздела обычных диэлектриков [38,39]. Рассмотрение более общей модели нелинейной среды не поменяет качественно полученный результат. Магнитоэлектрический эффект перемешает волны ТЕ и ТМ, что приведет к возникновению ненулевых всех трех компонент электрического поля поверхностной волны и спиновому моменту с нормальной и касательной компонентами.

Учет поглощения приведет к тому, что постоянная распространения будет комплексной величиной, и поверхностная волна будет затухать. Диэлектрические проницаемости, будучи комплексными величинами, сделают множитель $\rho$ в (10) или (14) комплексным. Разность фаз между $e_{y}(x)$ и $e_{z}(x)$ будет тогда отличаться от $\pi / 2$. Как 
видно из (8),

$g_{x}^{(1,2)} \sim|A||B| \sin \left(\psi_{1}-\psi_{2}\right), \quad g_{z}^{(1,2)} \sim|A||B| \cos \left(\psi_{1}-\psi_{2}\right)$,

вектор плотности спинового момента перестает быть поперечным вектором, но ненулевая нормальная компонента сохраняется.

\section{Финансирование работы}

Исследование выполнено при поддержке Российского фонда фундаментальных исследований (грант № 18-0200921).

\section{Список литературы}

[1] O'Neil A.T., MacVicar I., Allen L., Padgett M.J. // Phys. Rev. Lett. 2002. V. 88. P. 061101.

[2] Barnett S.M. // J. Mod. Opt. 2010. V. 57. N 14-15. P. 1339.

[3] Bialynicki-Birula I., Bialynicka-Birula Z. // J. Opt. 2011. V. 13. P. 064014.

[4] Barnett S.M., Allen L., Cameron R.P., Gilson C.R., Padgett M.J., Speirits F.C., Yao A.M. // J. Opt. 2016. V. 18. P. 064004 (12 pp).

[5] Chun-Fang Li // Phys. Rev. A. 2009. V. 80. P. 063814.

[6] Tamir T. (Ed.) / Integrated Optics. Berlin: Springer, 1983; M.: Mir, 1978.

[7] Aiello A., Marquardt Ch., Leuchs G. // Phys. Rev. A. 2010. V. 81. P. 053838.

[8] Bliokh K.Y., Bekshaev A.Y., Nori F. // arXiv:1308.0547 [physics. optics]

[9] Bliokh K.Y., Nori F. // Phys. Rev. A. 2012. V. 85. P. 061801(R).

[10] Bliokh K.Y., Nori F. // Phys. Rep. 2015. V. 592. P. 1-38.

[11] Bliokh K.Y., Bekshaev A.Y., Nori F. // New J. Phys. 2017. V. 19. P. 063021.

[12] Berini P. // Adv. Opt. Photon. 2009. V. 1. N 3. P. 484.

[13] Wang Y., Plummer E.W., Kempa K. // Adv. Phys. 2011. V. 60. N 5. P. 799.

[14] Silveirinha M., Engheta N. // Phys. Rev. B. 2007. V. 75. P. $075119(10$ p).

[15] Alekseyev L.V., Narimanov E.E., Tumkur T., Li H., Barnakov Yu.A., Noginov M.A. // Appl. Phys. Lett. 2010. V. 97. P. 131107.

[16] Konstantinidis K., Feresidis A.P. // J. Opt. 2015. V. 17. P. 105104

[17] Starko-Bowes R., Atkinson J., Newman W., Hu H., Kallos T., Palikaras G., Fedosejevs R., Pramanik S., Jacob Z. // J. Opt. Soc. Am. B. 2015. V. 32. N 10. P. 2074.

[18] Alam M.Z., De Leon I., Boyd R.W. // Science. 2016. V. 352. P. 795-797.

[19] Vezzoli St., Bruno V., DeVault Cl., Roger Th., Shalaev V.M., Boltasseva A., Ferrera M., Clerici M., Dubietis A., Faccio D. // arXiv:1709.06972v1 [physics.optics], Phys. Rev. Lett. 2018. V. 120. P. 043902.

[20] Elser J., Wangberg R., Podolskiy V.A., Narimanov E.E. // Appl. Phys. Lett. 2006. V. 89. P. 261102.

[21] Drachev V.P., Podolskiy V.A., Kildishev A.V. // Opt. Expr. 2013. V. 21. N 12. P. 15048.

[22] Hasan M.Z., Kane C.L. // Rev. Mod. Phys. 2010. V. 82. P. 3045.
[23] Xiao-Liang Qi, Shou-Cheng Zhang // Rev. Mod. Phys. 2011. V. 83. P. 1057.

[24] Wang-Kong Tse, MacDonald A.H. // Phys. Rev. Lett. 2010. V. 105. P. 057401 (4 p).

[25] Karch A. // Phys. Rev. B. 2011. V. 83. P. 245432 (5 p).

[26] Huerta L. // Phys. Rev. D. 2016. V. 94. P. 125021.

[27] Ляшко Е.И., Маймистов А.И. // Опт. и спектр. 2016. T. 121. № 4. C. 671.

[28] Lyashko E.I., Maimistov A.I., Gabitov I.R. // arXiv: 1706.05951v1 [physics. optics].

[29] Маймистов А.И., Ляшко Е.И. // Известия РАН. Сер.физ. 2018. T. 82. № 1. С. 27.

[30] Husakou A., Herrmann J. // Phys. Rev. Lett. 2007. V. 99. P. 127402.

[31] Jahani S., Zhao H., Jacob Z. // Appl. Phys. Lett. 2018. V. 113. P. 021103.

[32] Chernov A.S., Skobov V.G. // Phys. Lett. A. 1995. V. 205. P. 81.

[33] Poliakov E.Y., Markel V.A., Shalaev V.M., Botet R. // Phys. Rev. B. 1998. V. 57. N 23. P. 14901.

[34] Krasavin A.V., MacDonald K.F., Schwanecke A.S., Zheludev N.I. // Appl. Phys. Lett. 2006. V. 89. P. 031118.

[35] Ginzburg P., Hayt A., Berkovich N., Orenstein M. // Opt. Lett. 2010. V. 35. P. 551.

[36] Davoyn A.R., Shadrirov I.V., Kivshar Yu.S. // Opt. Lett. 2011. V. 36. N 6. P. 930.

[37] Агранович В.М., Бабиченко В.С., Черняк В.Я. // Письма в ЖЭТФ. 1980. Т. 32. № 8. C. 532; JETP Lett. 1980. V. 32. N 8. P. 512.

[38] Michalache D., Mazilu D. // Appl. Phys. B. 1985. V. 37. N 2. P. 107.

[39] Michalache D., Mazilu D. // Appl. Phys. B. 1986. V. 41. N 2. P. 119. 